\title{
Performance of Ultra-Wideband Communications With Suboptimal Receivers in Multipath Channels
}

\author{
John D. Choi, Student Member, IEEE, and Wayne E. Stark, Fellow, IEEE
}

\begin{abstract}
The performance of a single-user ultra-wideband (UWB) communication system employing binary block-coded pulse-position modulation (PPM) and suboptimal receivers in multipath channels is considered. The receivers examined include a rake receiver with various diversity combining schemes and an autocorrelation receiver, which is used in conjunction with transmitted reference (TR) signaling. A general framework is provided for deriving the performance of these receivers in multipath channels corrupted by additive white Gaussian noise (AWGN). By employing previous measurements of indoor UWB channels, we obtain numerical results for several cases which illustrate the tradeoff between performance and receiver complexity.
\end{abstract}

Index Terms-Autocorrelation receiver, diversity combining, on-off keying (OOK), pulse-position modulation (PPM), rake receiver, transmitted reference (TR), ultra-wideband (UWB).

\section{INTRODUCTION}

$\mathbf{U}$ LTRA-WIDEBAND (UWB) communications involves the transmission of short pulses with a relatively large fractional bandwidth [1], [2]. More specifically, these pulses possess a $-10-\mathrm{dB}$ bandwidth which exceeds $500 \mathrm{MHz}$ or $20 \%$ of their center frequency [3] and is typically on the order of one to several gigahertz. The large bandwidth occupancy of UWB signals primarily accounts for both the advantages and disadvantages associated with UWB communication systems [4], [5]. For instance, the large bandwidth of UWB signals in conjunction with appropriate spreading techniques [5]-[13] provides robustness to jamming, as well as a low probability of intercept and detection. These favorable characteristics are offset by the fact that UWB communication systems must coexist with narrowband and wideband systems already operating in dedicated frequency bands. In order to minimize interference to these systems, UWB systems must follow strict regulations [3] which limit the achievable data rates [4], [14], transmission range, and implementation of power control. The presence of multiple interfering signals also necessitates additional receiver complexity, even with the potentially large processing gains of UWB spread-spectrum (SS) systems.

This duality regarding UWB signaling also manifests itself in the effects of the channel. Because UWB signals possess such

Manuscript received December 21, 2001; revised August 27, 2002. This work was supported in part by the Office of Naval Research under Grant N00014-00-1-0224 and in part by the United States Army Research Office under Grant DAAH04-96-1-0377. This paper was presented in part at the International Conference on Communications (ICC), New York, NY, April 28-May 2, 2002 and in part at the Ultra-Wideband Systems and Technologies Conference (UWBST), Baltimore, MD, May 20-May 23, 2002.

The authors are with the Department of Electrical Engineering and Computer Science, University of Michigan, Ann Arbor, MI 48105 USA (e-mail: jdchoi@eecs.umich.edu; stark@eecs.umich.edu).

Digital Object Identifier 10.1109/JSAC.2002.805623 a large bandwidth, the channel is extremely frequency-selective and the received signal contains a significant number of resolvable multipath components [15]-[19]. The fine time resolution of UWB signals reduces the fading caused by several multipath components from different propagation paths overlapping in time and adding destructively [20]. However, each multipath component (or more appropriately, pulse [23]) associated with a particular path collectively exhibits distortion after reflections, diffractions, and scattering and does not resemble the ideal received signal corresponding to the line-of-sight (LOS) path [1], [2], [21]-[23]. This heightened sensitivity of UWB signals to different scatterers makes them particularly well-suited for radar applications [1], [2], [22], while making it more difficult for practical communications receivers to fully exploit the multipath diversity inherent in the received signal [16].

Among the main claimed advantages of UWB communication systems is the availability of technology to implement low-cost transceivers which can operate over such large bandwidths [5], [24]. In general, current embodiments of UWB receivers [4], [25], [26] sacrifice performance for low-complexity operation and a large discrepancy in performance exists between these implementations and the theoretically optimal receiver for most indoor and outdoor environments.

The most common receiver implementations cited in UWB literature include threshold detectors [4], [24], [26], correlation or rake receivers [1], [4]-[8], [10], [16]-[19], [21], [27]-[31], and autocorrelation receivers [1], [9], [11], [22], [32]. The relative performance of these receivers in multipath and jamming channels and the inherent tradeoff between performance and complexity have not been fully examined.

The majority of the performance analyses of UWB communication systems assumes the use of correlation receivers or rake receivers [10], [16]-[19], [21], [27]-[31]. Because of the large number of resolvable multipath components present in the received signal, practical UWB rake receivers must select, process, and combine only a small subset of these components (hence, employing hybrid selection combining (H-SC) [33]-[35] or suboptimal variations). The energy capture of UWB rake receivers can be relatively low for a moderate number of fingers [16] and is highly dependent upon the choice of tap delays. This selection takes on increased significance because UWB receivers generally do not employ a local oscillator [36] nor do they explicitly perform phase estimation and compensation in the demodulation process for a given tap delay.

An alternative approach to exploiting the multipath diversity is the use of an autocorrelation receiver which correlates the received signal with a previously received signal [1], [9], [11], [22], [32]. This receiver can capture the entire received 
signal energy for slowly varying channels without requiring channel estimation. The primary drawback of autocorrelation receivers, however, is the performance degradation associated with employing noisy received signals as reference signals in demodulation [37], [38]. Autocorrelation receivers have been typically used as suboptimal differential detectors for differential phase-shift keying (DPSK) in narrowband systems [37], [39]-[43]. The application of autocorrelation receivers to UWB systems was proposed for radar target detection purposes in [22]. The signaling and detection scheme described in this work [22] falls under the classification of a transmitted reference (TR) system [38]. TR communication systems operate by transmitting a pair of unmodulated and modulated signals and employing the former to demodulate the latter [9], [38]. Similar to differential modulation and pilot symbol-assisted modulation schemes, TR systems in essence transmit reference signals to generate side information regarding the channel. A TR-SS communication system employing UWB pulses or noise for signaling and a hybrid of time-hopping (TH) and direct-sequence (DS) spreading techniques has been recently proposed in [9], [11], [32].

This paper examines the performance of rake and autocorrelation receivers with varying degrees of complexity. We consider these receivers in the context of a single-user UWB system employing binary block-coded pulse-position modulation (PPM) in multipath channels corrupted by additive white Gaussian noise (AWGN). We extend previous work involving block-coded PPM and rake reception [8], [17], [21], [27], [28], [30] by providing a more general analytical framework and considering various suboptimal diversity combining schemes. These schemes select a subset of the received multipath components, either optimally or suboptimally, and combine them with maximal ratio combining (MRC) or square-law combining (SLC). Furthermore, we examine a TR system employing block-coded PPM and an autocorrelation receiver which averages previously received reference pulses as a means of noise suppression. It is noted that although UWB systems encounter narrowband and wideband jamming, the effect of such interference upon the system performance is not taken into consideration for analytical simplicity. In order to obtain numerical results, the performance of both the rake and autocorrelation receivers is evaluated with measured indoor channel data [15].

The paper is organized as follows. In Section II, the system model and performance analysis involving rake receivers are presented. Likewise, Section III details the performance analysis associated with the TR system. The performance of these two systems is evaluated for various cases by employing indoor UWB channel measurements in Section V.

\section{Performance of RAKe Receivers}

In this section, we consider a single-user UWB system employing binary block-coded PPM in a multipath channel with AWGN. We first describe the system model and then analyze the performance of a rake receiver with arbitrary tap delays and either MRC or SLC. Because of the assumptions made in this section, the results are independent of any $\mathrm{TH}$ which might be employed to help smoothen the power spectral density of the transmitted signal [44] or in a SS multiple-access system. It is also noted that some of the assumptions stated in the system model will apply to the TR system considered in Section III.

\section{A. System Model}

The user employs binary signaling in which the transmitted signals consist of a low duty cycle sequence of $N_{p}$ UWB pulses $p(t)$ with energy $E_{p}$. The signal waveforms correspond to code words of a binary block code with length and Hamming distance $N_{p}$ in which each code element is pulse-position modulated. The signals $\left\{s_{i}(t)\right\}_{i=0}^{1}$ are equally likely to be transmitted and are given by

$$
\begin{aligned}
s_{i}(t)=\sum_{j=0}^{N_{p}-1} \sqrt{E_{p}} \tilde{p}\left(t-j T_{f}-c_{i, j} \tau_{p}\right) & \\
& 0 \leq t \leq N_{p} T_{f}
\end{aligned}
$$

where $\tilde{p}(t)=p(t) / \sqrt{E_{p}}$ denotes a unit energy pulse with time duration $T_{p}, T_{f}$ is the average pulse repetition period $\left(T_{f} \gg\right.$ $\left.T_{p}\right), c_{i, j}$ is the $j$ th binary code element associated with the $i$ th code word being modulated, and $\tau_{p}$ is the delay associated with PPM. In general, overlapped PPM refers to the case in which $\tau_{p}<T_{p}$, while orthogonal PPM corresponds to the case in which $\tau_{p} \geq T_{p}$.

In order to simplify the ensuing analysis, the modulated code words are assumed to have equal weight, thereby implying that $N_{p}$ is even. For concreteness, the binary code elements are defined to be $c_{0, j}=j \bmod 2$ and $c_{1, j}=1-c_{0, j}, \forall j$. In addition, the cumulative effect of the transmit and receive antennas has been implicitly incorporated into the definition of $p(t)$ for notational simplicity.

The multipath channel is modeled as a linear, randomly time-varying filter which is time-invariant over a symbol duration with impulse response $h(t)$ and maximum excess delay spread $T_{m}\left(T_{m} \gg T_{p}\right)$. A tapped-delay-line representation of the channel impulse response is assumed with a tap spacing much less than $T_{p}$ and random tap weights. Furthermore, the pulse repetition period is chosen to be sufficiently large $\left(T_{f} \geq T_{m}+T_{p}+\tau_{p}\right)$ to preclude intersymbol and intrasymbol interference.

Assuming without any loss of generality that $s_{0}(t)$ is transmitted, the received signal is

$$
\begin{aligned}
r(t) & =s_{0}(t) * h(t)+n(t), \quad 0 \leq t \leq N_{p} T_{f} \\
& =\sum_{j=0}^{N_{p}-1} \sqrt{E_{p}} \tilde{p}\left(t-j T_{f}-c_{0, j} \tau_{p}\right) * h(t)+n(t)
\end{aligned}
$$

where $n(t)$ is a zero mean, AWGN random process with twosided power spectral density $N_{0} / 2$. The substitution of (1) into (2) yields the last expression (3). This expression can be simplified by employing the relation $g(t)=\tilde{p}(t) * h(t)$ as follows:

$$
r(t)=\sum_{j=0}^{N_{p}-1} \sqrt{E_{p}} g\left(t-j T_{f}-c_{0, j} \tau_{p}\right)+n(t) .
$$

Thus, the received signal energy in the absence of noise when conditioned upon the channel is $N_{p} E_{p} E_{g}$, where 
$E_{g}=\int_{0}^{T_{g}} g^{2}(t) d t$ and $T_{g}$ is the time duration of a received UWB pulse $\left(T_{g}=T_{m}+T_{p}\right)$.

Because UWB signals occupy such a large bandwidth, the channel is extremely frequency-selective. The received signal consequently contains a significant number of resolvable multipath components and in order to exploit the multipath diversity, a rake receiver is considered. Because of complexity constraints, however, the rake receiver processes only a subset of the total number of received multipath components. The receiver operates by passing $r(t)$ through a tapped-delay-line and performing cross correlations with two reference signals at $L$ tap delays $\left\{\tau_{l}\right\}_{l=0}^{L-1}$, where $0 \leq \tau_{0}<\tau_{1}<\cdots<\tau_{L-1} \leq T_{m}$. The normalized reference signals $\left\{\tilde{s}_{i}(t)\right\}_{i=0}^{1}$ corresponding to the two possible transmitted signals are given by

$\tilde{s}_{i}(t)=\frac{1}{\sqrt{N_{p}}} \sum_{j=0}^{N_{p}-1} \tilde{p}\left(t-j T_{f}-c_{i, j} \tau_{p}\right)$,

$$
0 \leq t \leq N_{p} T_{f}
$$

In general, the template pulse comprising the reference signals need not be equivalent to $\tilde{p}(t)$ because of either limitations in receiver complexity or the fact that UWB pulses which are reflected, diffracted, or scattered during propagation may not resemble the ideal received pulse corresponding to the LOS path [1], [2], [21], [22], [45]. The use of an orthonormal set of template pulses has been proposed in [16], [18], and [19] without accompanying performance results.

The operation of the rake receiver can be viewed from an alternative implementation in which the received signal is correlated with delayed versions of the reference signals [46]. By assuming that the tap delays are chosen such that $\left|\tau_{l}-\tau_{k}\right| \geq$ $T_{p}, \forall l \neq k$, the reference signals comprising $\left\{\tilde{s}_{0}\left(t-\tau_{l}\right)\right\}_{l=0}^{L-1}$ and $\left\{\tilde{s}_{1}\left(t-\tau_{l}\right)\right\}_{l=0}^{L-1}$, respectively, form two separate sets of orthonormal basis functions. The output of the correlator corresponding to the $l$ th finger of the rake receiver is given by

$Z_{i, l}=\int_{-\infty}^{\infty} r(t) \tilde{s}_{i}\left(t-\tau_{l}\right) d t$

$$
i=0,1, \quad l=0, \ldots, L-1 .
$$

Prior to evaluating the equation for $Z_{i, l}$, we define the crosscorrelation function between $g(t)$ and $\tilde{p}(t)$ as

$$
\alpha_{g \tilde{p}}(\tau)=\int_{-\infty}^{\infty} g(t) \tilde{p}(t-\tau) d t
$$

where $\alpha_{g \tilde{p}}(\tau)=0$ if $\tau \leq-T_{p}$ or $\tau \geq T_{g}$. The cross-correlation function is simply denoted as $\alpha(\tau)$ in the remainder of the paper for notational simplicity.

By substituting (4) and (5) into (6) and employing the previous definition (7), the output of the correlator becomes

$$
Z_{i, l}= \begin{cases}\sqrt{N_{p} E_{p}} \alpha\left(\tau_{l}\right)+\eta_{0, l}, & i=0 \\ \sqrt{N_{p} E_{p}} \frac{\alpha\left(\tau_{l}+\tau_{p}\right)+\alpha\left(\tau_{l}-\tau_{p}\right)}{2}+\eta_{1, l}, & i=1\end{cases}
$$

where $\eta_{i, l}=\int_{-\infty}^{\infty} n(t) \tilde{s}_{i}\left(t-\tau_{l}\right) d t$. The assumption that the modulated code words have equal weight is used in the derivation of $Z_{1, l}$ in (8). It is noted that $\left\{\eta_{0, l}\right\}_{l=0}^{L-1}$ and $\left\{\eta_{1, l}\right\}_{l=0}^{L-1}$ represent two separate sets of independent, zero-mean Gaussian random variables with variance $N_{0} / 2$. Collectively, these two sets of random variables are correlated or uncorrelated depending upon $\tau_{p}$.
Because the diversity combining schemes of interest manipulate these correlator outputs differently, their operation and associated performance are described in the ensuing two subsections.

\section{B. $M R C$}

The optimal linear combining technique is MRC which yields the maximum output signal-to-noise ratio (SNR) [46], [47]. For the rake receiver under consideration, the combiner appropriately weights the correlator outputs according to their SNR prior to summing. The performance and optimality of MRC consequently depend upon the receiver's knowledge of the channel. Assuming that the receiver can perfectly estimate these optimal weights, the maximal ratio combiner output corresponding to each decision hypothesis is

$$
Z_{i}=\sum_{l=0}^{L-1} \sqrt{N_{p} E_{p}} \alpha\left(\tau_{l}\right) Z_{i, l}, \quad i=0,1 .
$$

The substitution of (8) into (9) then leads to the following expansion:

$$
Z_{i}= \begin{cases}N_{p} E_{p} \sum_{l=0}^{L-1} \alpha^{2}\left(\tau_{l}\right)+\eta_{0}, & i=0 \\ N_{p} E_{p} \sum_{l=0}^{L-1} \alpha\left(\tau_{l}\right) \beta\left(\tau_{l}, \tau_{p}\right)+\eta_{1}, & i=1\end{cases}
$$

where $\eta_{i}=\sqrt{N_{p} E_{p}} \sum_{l=0}^{L-1} \alpha\left(\tau_{l}\right) \eta_{i, l}$ and $\beta\left(\tau_{l}, \tau_{p}\right)=\left[\alpha\left(\tau_{l}+\right.\right.$ $\left.\left.\tau_{p}\right)+\alpha\left(\tau_{l}-\tau_{p}\right)\right] / 2$.

For notational simplicity, we collectively denote the channeldependent random variables $\left\{\alpha\left(\tau_{l}\right), \beta\left(\tau_{l}, \tau_{p}\right)\right\}_{l=0}^{L-1}$ as $\underline{U}$ in this section. By conditioning upon $\underline{U}$, it can be shown that the decision statistics $\left\{Z_{i}\right\}_{i=0}^{1}$ are jointly Gaussian random variables which are either correlated or independent depending on $\tau_{p}$. In order to derive the conditional probability of error, we define the random variable $Z_{\Delta}=Z_{0}-Z_{1}$ which is conditionally Gaussian with the following mean and variance:

$$
\begin{aligned}
E\left[Z_{\Delta} \mid \underline{U}=\underline{u}\right] & =N_{p} E_{p} \sum_{l=0}^{L-1} \alpha^{2}\left(\tau_{l}\right)-\alpha\left(\tau_{l}\right) \beta\left(\tau_{l}, \tau_{p}\right) \\
\operatorname{Var}\left(Z_{\Delta} \mid \underline{U}=\underline{u}\right) & =N_{p} E_{p} N_{0}\left[\left(1-\gamma_{p}\left(\tau_{p}\right)\right) \sum_{l=0}^{L-1} \alpha^{2}\left(\tau_{l}\right)\right. \\
& \left.-\sum_{l=0}^{L-1} \sum_{\substack{k=0 \\
k \neq l}}^{L-1} \alpha\left(\tau_{l}\right) \alpha\left(\tau_{k}\right) \cdot \gamma_{p}\left(\tau_{l}-\tau_{k}+\tau_{p}\right)\right] .
\end{aligned}
$$

In (12), $\gamma_{p}(\tau)$ denotes the normalized autocorrelation function of $p(t)$ and is given by

$$
\gamma_{p}(\tau)=\int_{-\infty}^{\infty} \tilde{p}(t) \tilde{p}(t-\tau) d t
$$

From (11) and (12), the conditional probability of error is simply

$$
\begin{aligned}
P_{e}(\underline{u}) & =P\left[Z_{\Delta}<0 \mid \underline{U}=\underline{u}, s_{0}(t) \text { transmitted }\right] \\
& =Q\left(\sqrt{\frac{E\left[Z_{\Delta} \mid \underline{U}=\underline{u}\right]^{2}}{\operatorname{Var}\left(Z_{\Delta} \mid \underline{U}=\underline{u}\right)}}\right)
\end{aligned}
$$


where $Q(x)=1 / \sqrt{2 \pi} \int_{x}^{\infty} e^{-t^{2} / 2} d t$. As evidenced by the derivations for the conditional mean and variance of $Z_{\Delta}$, the resulting equation for the conditional error probability for binary block-coded PPM is cumbersome in general.

It is noted that the term $\sum_{l=0}^{L-1} \alpha^{2}\left(\tau_{l}\right)$ in (11) and (12) denotes the energy capture of the rake receiver. The normalized energy capture $\sum_{l=0}^{L-1} \alpha^{2}\left(\tau_{l}\right) / E_{g}$, where $E_{g}$ is defined in (4), is simply the normalized energy of the signal space representation of the noiseless received signal. This notion of energy capture is a loose interpretation of the definition provided in [16] which specifies the tap delays $\left\{\tau_{l}\right\}_{l=0}^{L-1}$ to be optimal. The impact of selecting optimal, as well as suboptimal, delays upon the performance of rake receivers is discussed in Section IV. In addition, the normalized energy capture equals one when the noiseless received signal can be expressed completely in terms of one of the two sets of orthonormal basis functions and corresponds to the optimal receiver for the case of MRC.

Although the PPM delay $\tau_{p}$ is typically chosen to be less than one or a few multiples of $T_{p}$, we consider the case in which the two possible received signals without noise are orthogonal $\left(\tau_{p}=T_{g}\right)$. Substituting this value of $\tau_{p}$ into (15) and noting that $\gamma_{p}(\tau)=0$ for $|\tau| \geq T_{p}$, we obtain the following simplification:

$$
P_{e}(\underline{u})=Q\left(\sqrt{\frac{N_{p} E_{p} \sum_{l=0}^{L-1} \alpha^{2}\left(\tau_{l}\right)}{N_{0}}}\right) .
$$

It is noted that this particular case of block-coded orthogonal PPM is equivalent to one in which the code elements of a different set of equal weight code words of length and Hamming distance $N_{p}$ are modulated by on-off keying (OOK) with a pulse repetition period of $T_{f}=T_{g}$.

\section{C. $S L C$}

A suboptimal, reduced-complexity diversity combining scheme is SLC which does not require an estimate of the optimal weights. This scheme is commonly employed in conjunction with orthogonal modulation and noncoherent reception in wideband and multichannel systems. For the previously described rake receiver structure, the square-law combiner operates by squaring and then summing the correlator outputs $Z_{i, l}$ associated with each possible transmitted signal. The decision statistics after SLC are thus

$$
Z_{i}=\sum_{l=0}^{L-1} Z_{i, l}^{2}, \quad i=0,1 .
$$

In general, SLC is not well-suited for overlapped PPM and must be used with orthogonal PPM $\left(\tau_{p} \geq T_{p}\right)$. In order to exploit the multipath diversity of the received signal and obtain reasonable performance, the delay $\tau_{p}$ is chosen such that $\tau_{p} \geq \tau_{L-1}+T_{p}$.

The substitution of the correlator outputs (8) into (17) yields the following:

$$
Z_{i}= \begin{cases}\sum_{l=0}^{L-1}\left(\sqrt{N_{p} E_{p}} \alpha\left(\tau_{l}\right)+\eta_{0, l}\right)^{2}, & i=0 \\ \sum_{l=0}^{L-1}\left(\sqrt{N_{p} E_{p}} \frac{\alpha\left(\tau_{l}+\tau_{p}\right)}{2}+\eta_{1, l}\right)^{2}, & i=1\end{cases}
$$

where the simplification for $Z_{1}$ results from the fact that for the $\tau_{p}$ being considered, $\alpha\left(\tau_{l}-\tau_{p}\right)=0, \forall l$.

Conditioned upon $\underline{U}$, the decision statistics are both independent noncentral chi-square random variables with $L$ degrees of freedom for $\tau_{L-1}+T_{p} \leq \tau_{p}<T_{g}$, whereas $Z_{1}$ becomes a central chi-square random variable for $\tau_{p} \geq T_{g}$. Although the conditional probability of error can be derived for these two cases (see Appendix A), the resulting equations do not provide much insight into the relative performance of SLC with respect to MRC.

As a result, we consider the case in which the number of combined multipath components $(L)$ grows large and the decision statistics in (18) can be modeled as independent Gaussian random variables when conditioned upon $\underline{U}$. Similar to the case of MRC, we define the conditionally Gaussian random variable $Z_{\Delta}=Z_{0}-Z_{1}$, which possesses the following mean and variance:

$$
\begin{gathered}
E\left[Z_{\Delta} \mid \underline{U}=\underline{u}\right]=N_{p} E_{p} \sum_{l=0}^{L-1} \alpha^{2}\left(\tau_{l}\right)-\frac{\alpha^{2}\left(\tau_{l}+\tau_{p}\right)}{4} \\
\operatorname{Var}\left(Z_{\Delta} \mid \underline{U}=\underline{u}\right)=N_{p} E_{p} N_{0}\left[\frac{L N_{0}}{N_{p} E_{p}}+2 \sum_{l=0}^{L-1} \alpha^{2}\left(\tau_{l}\right)\right. \\
\left.+\frac{\alpha^{2}\left(\tau_{l}+\tau_{p}\right)}{4}\right]
\end{gathered}
$$

Thus, the approximate conditional error probability of SLC for the case of large $L$ is obtained by simply substituting the above equations into the previous derivation (15). As might be expected, the energy capture parameter is present in $P_{e}(\underline{u})$ for SLC through (19) and (20).

Furthermore, we note that the first term of the conditional variance (20) corresponds to the noise-on-noise term which occurs when expanding the equations in (18). This term degrades the performance of the rake receiver with SLC whenever the received SNR is small relative to $L$. It is emphasized that prior to making a comparison between SLC and MRC or any two schemes, the potential differences in data rates arising from the selection of $\tau_{p}$ must be taken into account.

The performance gap between SLC and MRC is more clearly illustrated by again considering the case in which $\tau_{p}=T_{g}$. For this designation, the conditional mean and variance of $Z_{\Delta}$ simplify because $\alpha\left(\tau_{l}+\tau_{p}\right)=0, \forall l$, in (19) and (20). The conditional probability of error under the Gaussian approximation then becomes

$$
P_{e}(\underline{u})=Q\left(\sqrt{\frac{N_{p} E_{p} \sum_{l=0}^{L-1} \alpha^{2}\left(\tau_{l}\right)}{N_{0}\left[2+\left(\frac{N_{p} E_{p} \sum_{l=0}^{L-1} \alpha^{2}\left(\tau_{l}\right)}{L N_{0}}\right)^{-1}\right]}}\right) .
$$

This equation indicates that binary block-coded OOK with SLC suffers a 3-dB loss relative to the same signaling scheme with MRC (16) when the Gaussian approximation is valid and the SNR is very large. The noise-on-noise term manifests in the second term of the denominator in (21) and has a deleterious effect for low to moderate SNR.

\section{Performance of TR System With AUTOCORRELATION RECEIVER}

An alternative approach to collecting the received multipath signal energy is the use of autocorrelation receivers which 
perform differential detection suboptimally. In this section, we consider a TR system employing binary block-coded PPM and an autocorrelation receiver which suppresses the noise by averaging the reference pulses prior to demodulation. The analysis below does not take into consideration the effect of TH for simplicity.

\section{A. System Model}

The user employs a variation of the binary signaling scheme described in Section II. In particular, the TR system transmits a reference pulse which carries no information prior to each pulse-position modulated data pulse. The modulated data pulses correspond to code elements of equal weight, binary code words of length and Hamming distance $N_{p} / 2$ (thereby implying that $N_{p} / 2$ is even). The signals $\left\{s_{i}(t)\right\}_{i=0}^{1}$ are equally likely to be transmitted and are given by

$$
s_{i}(t)=\sum_{j=0}^{N_{p} / 2-1} \sqrt{E_{p}} \tilde{p}\left(t-j T_{f}\right)+\sqrt{E_{p}} \tilde{p}\left(t-j T_{f}-\tau_{i, j}\right)
$$

where $t \in\left[0, N_{p} T_{f} / 2\right], T_{f}=2\left(T_{m}+T_{p}\right)+\tau_{p}$, and $\tau_{i, j}=$ $T_{m}+T_{p}+c_{i, j} \tau_{p}$. Unless otherwise stated, the previous assumptions regarding the parameters in (22) still hold. In addition, the user is assumed to have been transmitting continuously since the previous symbol duration.

We employ the same channel model as before with the notable exception being that the channel is assumed to be timeinvariant over two symbol durations, as opposed to a single symbol duration.

Assuming without any loss of generality that $s_{0}(t)$ is transmitted, the received signal is

$$
\begin{aligned}
r(t)= & s_{0}(t) * h(t)+n(t) \\
= & \sum_{j=0}^{N_{p} / 2-1} \sqrt{E_{p}} g\left(t-j T_{f}\right)+\sqrt{E_{p}} g\left(t-j T_{f}-\tau_{0, j}\right) \\
& +n(t)
\end{aligned}
$$

where $t \in\left[0, N_{p} T_{f} / 2\right]$ and (24) employs the substitution of (22) into (23), as well as the same relation specified in (4). We also define $T_{g}=T_{m}+T_{p}$ and $E_{g}=\int_{0}^{T_{g}} g^{2}(t) d t$ as before in (4).

The autocorrelation receiver under consideration first passes $r(t)$ through an ideal bandpass filter with bandwidth $W$ and center frequency $f_{c}$. The bandwidth of the filter is chosen to be sufficiently wide $\left(W \gg 1 / T_{g}\right)$ such that negligible intersymbol and intrasymbol interference results [37]. The received signal after filtering is expressed as

$$
\begin{aligned}
\hat{r}(t)=\sum_{j=0}^{N_{p} / 2-1} \sqrt{E_{p}} \hat{g}( & \left.t-j T_{f}\right) \\
& +\sqrt{E_{p}} \hat{g}\left(t-j T_{f}-\tau_{0, j}\right)+\hat{n}(t)
\end{aligned}
$$

where $\hat{n}(t)$ is a zero mean, Gaussian random process. The autocorrelation function of the filtered noise [37] is given by

$$
R_{\hat{n}}(\tau)=E[\hat{n}(t) \hat{n}(t+\tau)]=N_{0} W \operatorname{sinc}(W \tau) \cos \left(2 \pi f_{c} \tau\right)
$$

where $\operatorname{sinc}(x)=\sin (\pi x) / \pi x$.
In order to demodulate the $j$ th data pulse, the receiver first multiplies $\hat{r}(t)$ during the time frame $t \in\left[j T_{f}+\tau_{i, j},(j+\right.$ 1) $\left.T_{f}\right]$ with an appropriately delayed average of the $N(1 \leq N \leq$ $N_{p} / 2$ ) previously received reference pulses. The receiver then integrates this product over time duration $T\left(0<T \leq T_{g}\right)$. The autocorrelator outputs corresponding to the two possible transmitted code elements for the $j$ th received data pulse are thus

$$
Z_{i, j}=\int_{j T_{f}+\tau_{i, j}}^{j T_{f}+\tau_{i, j}+T} \hat{r}(t)\left[\frac{1}{N} \sum_{k=0}^{N-1} \hat{r}\left(t-k T_{f}-\tau_{i, j}\right)\right] d t
$$

where the term inside the brackets corresponds to the averaged reference pulses. Next, by making the substitution $v=t-$ $\left(j T_{f}+\tau_{i, j}\right)$ and taking into account the previously received symbol $\left(t \in\left[-N_{p} T_{f} / 2,0\right]\right)$, the outputs can be reexpressed as

$$
\begin{aligned}
& Z_{i, j}= \int_{0}^{T}\left[\sqrt{E_{p}} \hat{g}\left(v+\tau_{i, j}-\tau_{0, j}\right)+\hat{n}\left(v+j T_{f}+\tau_{i, j}\right)\right] \\
& \cdot\left[\frac{1}{N} \sum_{k=0}^{N-1} \sqrt{E_{p}} \hat{g}(v)+\hat{n}\left(v+(j-k) T_{f}\right)\right] d v
\end{aligned}
$$

We next define $Z_{\Delta, j}=Z_{0, j}-Z_{1, j}$ and employ the previous result (28) to obtain the following expansion:

$$
Z_{\Delta, j}=X_{1, j}+X_{2, j}+\sum_{k=0}^{N-1} X_{3, j, k}+\sum_{k=0}^{N-1} X_{4, j, k}
$$

where

$$
\begin{aligned}
X_{1, j}= & E_{p} \int_{0}^{T}\left[\hat{g}(t)-\hat{g}\left(t+\tau_{1, j}-\tau_{0, j}\right)\right] \hat{g}(t) d t \\
X_{2, j}= & \sqrt{E_{p}} \int_{0}^{T}\left[\hat{n}\left(t+j T_{f}+\tau_{0, j}\right)\right. \\
& \left.-\hat{n}\left(t+j T_{f}+\tau_{1, j}\right)\right] \cdot \hat{g}(t) d t \\
X_{3, j, k}= & \frac{\sqrt{E_{p}}}{N} \int_{0}^{T}\left[\hat{g}(t)-\hat{g}\left(t+\tau_{1, j}-\tau_{0, j}\right)\right] \\
& \cdot \hat{n}\left(t+(j-k) T_{f}\right) d t \\
X_{4, j, k}= & \frac{1}{N} \int_{0}^{T}\left[\hat{n}\left(t+j T_{f}+\tau_{0, j}\right)-\hat{n}\left(t+j T_{f}+\tau_{1, j}\right)\right] \\
& \cdot \hat{n}\left(t+(j-k) T_{f}\right) d t .
\end{aligned}
$$

We note that the last two terms in (29) reflect the performance degradation associated with correlating the received data pulse with averaged reference pulses which contain noise.

The decision statistic $Z_{\Delta}$ is obtained by summing over all of the differences between the autocorrelation outputs as follows:

$$
\begin{aligned}
Z_{\Delta} & =\sum_{j=0}^{N_{p} / 2-1} Z_{\Delta, j} \\
& =\sum_{j=0}^{N_{p} / 2-1}\left[X_{1, j}+X_{2, j}+\sum_{k=0}^{N-1} X_{3, j, k}+\sum_{k=0}^{N-1} X_{4, j, k}\right] \\
& =Y_{1}+Y_{2}+Y_{3}+Y_{4}
\end{aligned}
$$


where $Y_{1}, Y_{2}, Y_{3}$, and $Y_{4}$ in the last step correspond to the four quantities being summed in (35), respectively.

In this section, the random variables associated with the tapped-delay-line representation of $h(t)$ are collectively denoted as $\underline{U}$. Conditioned upon $\underline{U}$, the decision statistic (36) can be decomposed as follows: $Y_{1}$ is nonrandom, $Y_{2}$ is a sum of weakly correlated, jointly Gaussian random variables $\left\{X_{2, j}\right\}, Y_{3}$ is a sum of correlated, jointly Gaussian random variables $\left\{X_{3, j, k}\right\}$, and $Y_{4}$ is a zero mean random variable which is uncorrelated with $Y_{2}$ and $Y_{3}$ (see Appendix B).

It is noted that modeling equations similar to $\left\{X_{4, j, k}\right\}$ in (33), which comprise $Y_{4}$ (36), has proven to be problematic in the analysis of autocorrelation receivers for DPSK [37], [43]. By employing a truncated Fourier series expansion of the received signal (24) [37], [39]-[42] and conditioning upon $\underline{U}, X_{4, j, k}$ can be approximated as the difference of two correlated central chisquare random variables. If we interchange the summations corresponding to $Y_{4}$ and assume that $N_{p}$ is large, then for a given $k, \sum_{j=0}^{N_{p} / 2-1} X_{4, j, k}$ can be approximated as the difference of two correlated jointly Gaussian random variables. For the SNR range of general interest, the Gaussian approximation is also valid when $T W$ is large [37]. Consequently, we assume that $Y_{4}$ is a zero mean conditionally Gaussian random variable.

Furthermore, it is assumed for simplicity that $Y_{2}$ and $Y_{3}$, which are very weakly correlated when $W \gg 1 / T_{g}$, are uncorrelated. Thus, the decision statistic $Z_{\Delta}$ (36) when conditioned upon $\underline{U}$ is approximated as the sum of a constant, $Y_{1}$, and three mutually independent, zero-mean Gaussian random variables $\left\{Y_{2}, Y_{3}, Y_{4}\right\}$. As a result, $Z_{\Delta}$ itself is conditionally Gaussian with the following mean:

$$
E\left[Z_{\Delta} \mid \underline{U}=\underline{u}\right]=\frac{N_{p} E_{p}}{2}\left[\int_{0}^{T} \hat{g}^{2}(t) d t-\rho\left(\tau_{p}\right)\right]
$$

where $\rho\left(\tau_{p}\right)=(1 / 2) \int_{0}^{T} \hat{g}(t)\left[\hat{g}\left(t+\tau_{p}\right)+\hat{g}\left(t-\tau_{p}\right)\right] d t$. The first term inside the brackets (37) represents the energy capture of the autocorrelation receiver and approximately equals $E_{g}$ if $T=T_{g}$ and $W$ exceeds a sufficient bandwidth measure of $p(t)$.

For the case in which $N=N_{p} / 2$ previous reference pulses are averaged by the autocorrelation receiver, the conditional variance of the decision statistic is given by

$$
\operatorname{Var}\left(Z_{\Delta} \mid \underline{U}=\underline{u}\right) \simeq \frac{N_{p} E_{p} N_{0}}{2}\left[\zeta_{2}+\zeta_{3}+\frac{2 N_{0} T W \zeta_{4}}{N_{p} E_{p}}\right]
$$

where $\zeta_{2}, \zeta_{3}$, and $\zeta_{4}$ are associated with the conditional variance of $Y_{2}, Y_{3}$, and $Y_{4}$, respectively. These terms are fully specified in Appendix B and their dependence upon various parameters, including $\tau_{p}$, is suppressed for notational simplicity.

The conditional probability of error then immediately follows from the substitution of (37) and (38) into (15) and is given by

$$
P_{e}(\underline{u})=Q\left(\sqrt{\frac{N_{p} E_{p}\left[\int_{0}^{T} \hat{g}^{2}(t) d t-\rho\left(\tau_{p}\right)\right]^{2}}{2 N_{0}\left[\zeta_{2}+\zeta_{3}+\left(\frac{N_{p} E_{p}}{2 N_{0} T W \zeta_{4}}\right)^{-1}\right]}}\right) .
$$

We note that the third term in the denominator of (39) is asociated with the conditional variance of $Y_{4}$ and becomes negligible for large SNR, while degrading the system performance for low to moderate SNR or large $T W$. For the case in which the reference pulses are not averaged $(N=1)$, this term does not contain $N_{p}$ and consequently becomes large when the SNR of each received UWB pulse is small. Because the central limit theorem [46] was invoked in this section, as well as in the case of SLC, to approximate the sum of conditionally independent chi-square random variables, the corresponding conditional probability of error expressions exhibit several similarities and the product $T W$ can be interpreted as a measure of diversity order for the autocorrelation receiver.

As in the previous section, we consider the case in which $\tau_{p}=$ $T_{g}$ and the two possible received signals without noise and the reference pulses are orthogonal. This particular scheme can be equivalently viewed as block-coded OOK in which a reference pulse is transmitted for every two modulated code elements. By substituting this delay into (39), the conditional probability of error for $N=N_{p} / 2$ is given by

$$
P_{e}(\underline{u})=Q\left(\sqrt{\frac{N_{p} E_{p}\left[\int_{0}^{T} \hat{g}^{2}(t) d t\right]^{2}}{2 N_{0}\left[\zeta_{2}^{\prime}+\zeta_{3}^{\prime}+\left(\frac{N_{p} E_{p}}{2 N_{0} T W \zeta_{4}^{\prime}}\right)^{-1}\right]}}\right)
$$

where $\zeta_{2}^{\prime}, \zeta_{3}^{\prime}$, and $\zeta_{4}^{\prime}$ are defined in Appendix C. Equation (40) clearly parallels that for the case of block-coded OOK with rake reception and SLC (21).

\section{NumericAl RESUlTS}

In this section, the performances of block-coded overlapped PPM and OOK are compared for the different systems considered by employing measured channel data. In order to make a meaningful comparison, the parameters of the different schemes employing overlapped PPM and OOK are chosen appropriately such that they possess a date rate (bits/sec) equal to or approximately equal to $r=1 / N_{p} T_{g}$.

\section{A. Processing Measured Indoor Multipath Channel Data}

In order to obtain the numerical results, we use previous measurements of UWB signals received over short transmission distances (approximately 5.5-17.5 m) in a modern office building [15], [18]. The ideal received UWB signal associated with the LOS path resembles the third derivative of a Gaussian pulse in these measurements. A truncated and normalized version of the signal, which corresponds to $\tilde{p}(t)$ in (1), is illustrated in Fig. 1. This pulse possesses a $-10-\mathrm{dB}$ bandwidth of approximately $1.1 \mathrm{GHz}$, a center frequency of $1.1 \mathrm{GHz}$, and a time duration of $T_{p}=2 \mathrm{~ns}$.

We consider only $N_{c}=490$ of the 741 measurements, which were taken over a 300 ns window, and omit rooms $\mathrm{A}, \mathrm{B}, \mathrm{C}, \mathrm{E}, \mathrm{HW}$, and $\mathrm{R}$. The rooms $\mathrm{A}, \mathrm{B}, \mathrm{C}$, and $\mathrm{E}$ have particularly low SNR and the beginning of the received signal could not be easily distinguished from the noise floor. The majority of the channels in the measurement campaign have 


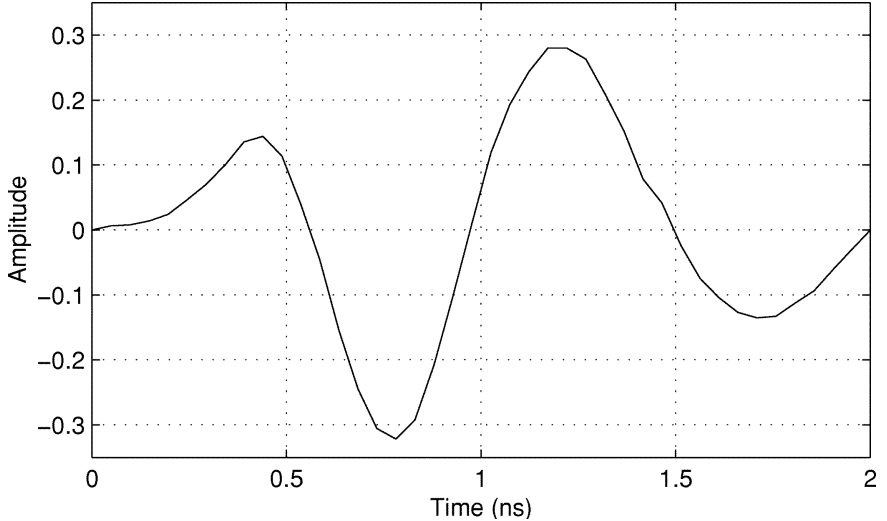

Fig. 1. The ideal received UWB pulse associated with the LOS path in the measured date.

an obstructed LOS propagation path (NLOS). As a result of shadowing and the different transmission distances, the channel data is normalized such that the average received energy for each set of measurements taken within a room is the same for all ten rooms considered. This modification attempts to isolate the deleterious effects of multipath fading from those of shadowing and path loss. The performance degradation resulting from these latter channel impairments is considered in [45] and [48]-[50].

The expected received energy per bit is approximated by the sample mean of the energy of the measured received signals [which corresponds to $E_{g}$ in (4)]. For overlapped PPM with rake reception, this quantity is given by

$$
\bar{E}_{b}=N_{p} E_{p} E\left[E_{g}\right] \simeq \frac{N_{p} E_{p}}{N_{c}} \sum_{i=0}^{N_{c}-1} E_{g}^{(i)}=N_{p} E_{p}
$$

where the superscript in $E_{g}^{(i)}$ indexes the channel realizations and the last equality results from the normalizations performed on the data set. The approximation in (41) must be appropriately modified for the other systems considered, as should the corresponding conditional error probability expressions, to reflect the reduced number of pulses which can be approximately transmitted during a symbol duration of $N_{p} T_{g}$.

As in [21], the expected probability of bit error is approximated by averaging over the channel realizations as follows:

$$
\begin{aligned}
P_{e} & =\int_{-\infty}^{\infty} \ldots \int_{-\infty}^{\infty} P_{e}(\underline{u}) f_{\underline{U}}(\underline{u}) d \underline{u} \\
& \simeq \frac{1}{N_{c}} \sum_{i=0}^{N_{c}-1} P_{e}\left(\underline{u}^{(i)}\right)
\end{aligned}
$$

where in (42) $f_{\underline{U}}(\underline{u})$ denotes the joint probability density function of the channel-dependent random variables and $P_{e}(\underline{u})$ corresponds to the previously derived conditional error probabilities.

\section{B. Optimal Receiver}

Prior to evaluating the performance of various suboptimal receivers, we first determine the performance of the optimal receiver for both block-coded overlapped PPM and OOK in

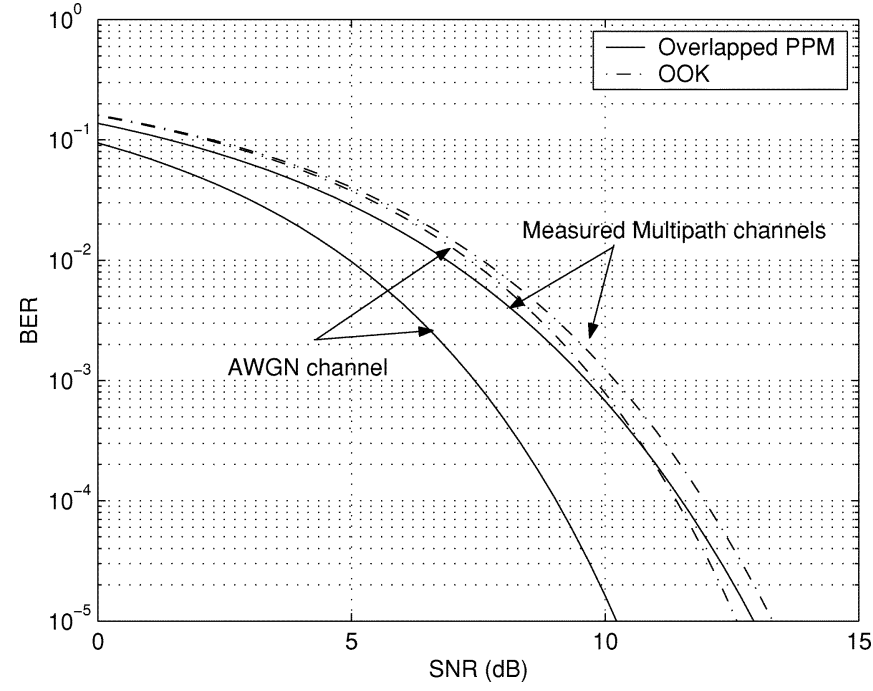

Fig. 2. Performance of the optimal receiver for binary block-coded overlapped PPM and OOK in the AWGN channel and the measured multipath channels.

the measured multipath channels. For overlapped PPM, the delay $\tau_{p}$ which minimizes the autocorrelation function of $\tilde{p}(t)$ and, hence, the probability of error in AWGN, is chosen $\left(\tau_{p}^{*}=0.4395 \mathrm{~ns}, \gamma_{p}\left(\tau_{p}^{*}\right)=-0.7268\right)$. This delay is used throughout this section when evaluating the performance of overlapped PPM.

Fig. 2 presents the performance of the two modulation schemes for the AWGN channel, as well as the measured multipath channels. In this and all subsequent figures, the average bit-error rate (BER) is plotted with respect to the average received SNR $\left(\bar{E}_{b} / N_{0}\right)$. We note that overlapped PPM provides a 2.39-dB gain over OOK in AWGN but only a 0.38-dB gain in the indoor multipath channels at $\mathrm{BER}=10^{-5}$. The performance degradation of overlapped PPM stems primarily from NLOS channels which cause the received signals to possess poor cross-correlation properties at $\tau_{p}^{*}$.

\section{Rake Receiver}

For the rake receiver structure considered in Section II, the choice of tap delays plays a significant role in the system performance. We first consider the case in which the $L$ selected delays are optimal and yield the maximum energy capture for a given channel realization. This selection scheme is identified as selection combining (SC), although SC is typically associated with the case where only the strongest path is employed in demodulation. Next, we consider two suboptimal, reduced-complexity selection schemes. The first of these suboptimal schemes, SC-NSC, couples SC with nonselective combining (NSC) in that only the strongest path is tracked $\left(\tau_{0}\right)$ and the remaining $L-1$ tap delays are chosen in a suboptimal manner such that $\tau_{l}=\tau_{0}+l T_{p}$, for $l=1, \ldots, L-1$. The third and final selection scheme considered is NSC, where the first $L$ arriving multipath components are chosen independent of their signal strength; that is, $\tau_{l}=l T_{p}, \forall l$ [30], [45].

The $L$ multipath components which are selected according to these schemes are then combined using MRC or SLC. Consequently, we consider a total of six different diversity combining schemes in this subsection: SC/MRC, 


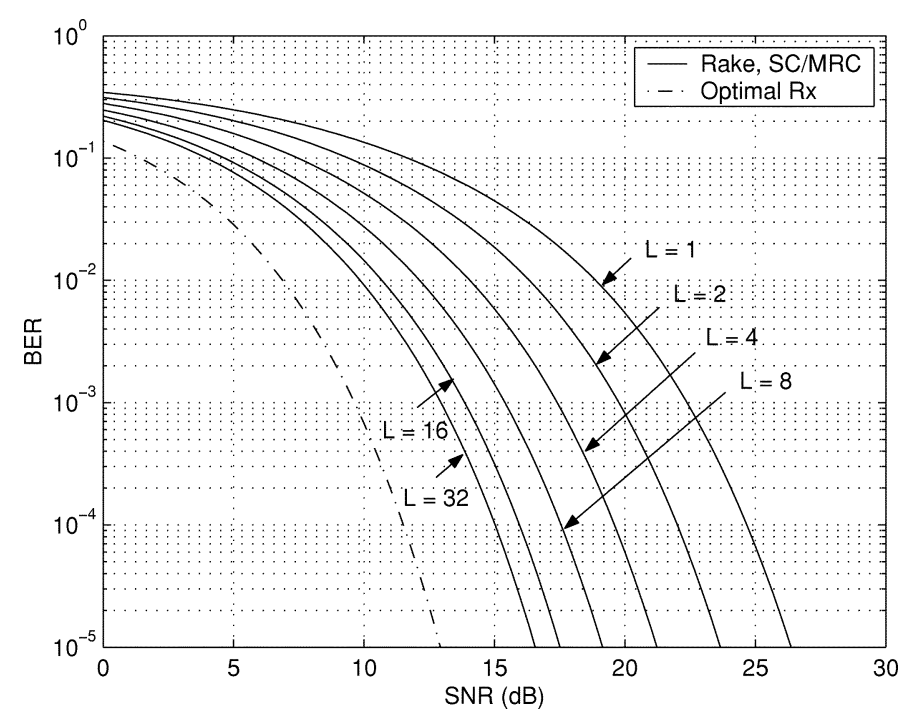

Fig. 3. Performance of binary block-coded overlapped PPM with rake reception and SC/MRC for $(L=1,2,4,8,16,32)$.

SC-NSC/MRC, NSC/MRC, SC/SLC, SC-NSC/SLC, and NSC/SLC, where SC/MRC and SC/SLC are also referred to as hybrid selection combining [33]-[35]. The performance of overlapped PPM is considered only in conjunction with schemes employing MRC, whereas the performance of OOK is considered for all of the cases.

We note that the first arriving path is typically not the strongest multipath component in NLOS channels [18], whereas the first arriving path is strong in LOS channels. As a result, SC-NSC is better suited than NSC for NLOS channels where the strongest path can be tracked, while both SC-NSC and NSC should perform adequately in LOS channels.

First, we consider the performance results for binary block-coded overlapped PPM with MRC and the different selection schemes. Fig. 3 illustrates the performance of overlapped PPM with SC/MRC in the measured multipath channels. This scheme yields the best performance among the diversity combiners considered while requiring the most complexity. The figure indicates that diminishing performance gains are achieved after $L=32$, at which point the scheme is $3.63-\mathrm{dB}$ removed from the performance of the optimal receiver at $\mathrm{BER}=10^{-5}$. The case of overlapped PPM with SC-NSC/MRC corresponds to Fig. 4, which shows that the scheme provides reduced gains relative to $\mathrm{SC} / \mathrm{MRC}$, as might be expected. In fact, the performance difference between selecting optimal delays and selecting one optimal and the rest suboptimal delays increases as the number of combined paths increases. For the case where $L=32$, SC-NSC/MRC performs $8.63-\mathrm{dB}$ worse than the optimal receiver at $\mathrm{BER}=10^{-5}$. Finally, Fig. 5 details the performance of overlapped PPM with NSC/MRC. Although the performance of NSC/MRC is comparable to that of SC-NSC/MRC for large diversity orders ( $L \geq 32$ ) (in part because most of the received signal energy arrives at the beginning), there exists a significant performance degradation for small $L$, thereby underscoring the importance of tap delay selection.

Next, the performance of binary block-coded OOK with rake reception and SLC is considered for the three different selec-

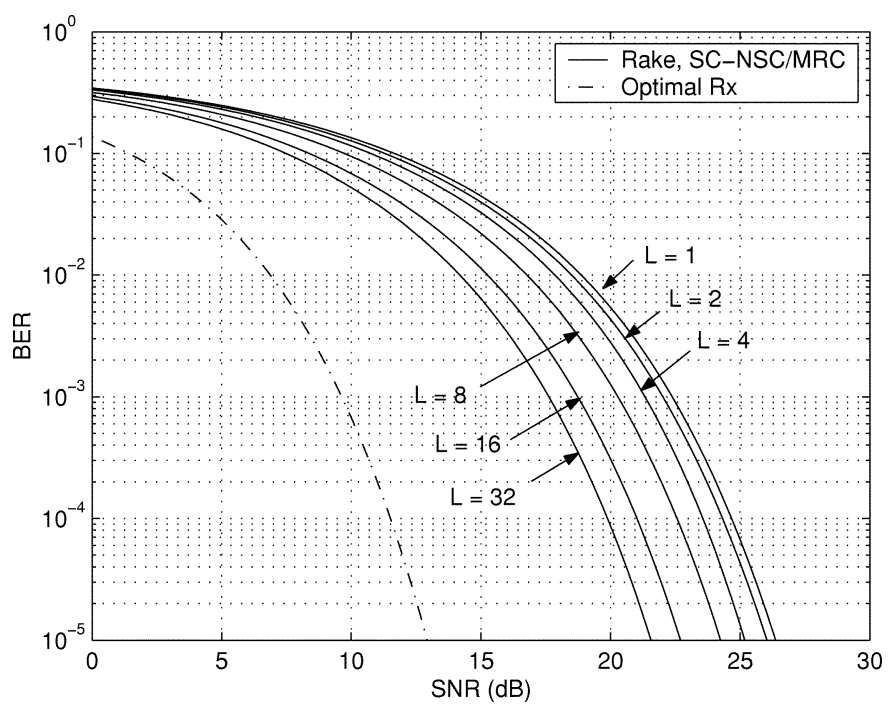

Fig. 4. Performance of binary block-coded overlapped PPM with rake reception and SC-NSC/MRC for $(L=1,2,4,8,16,32)$.

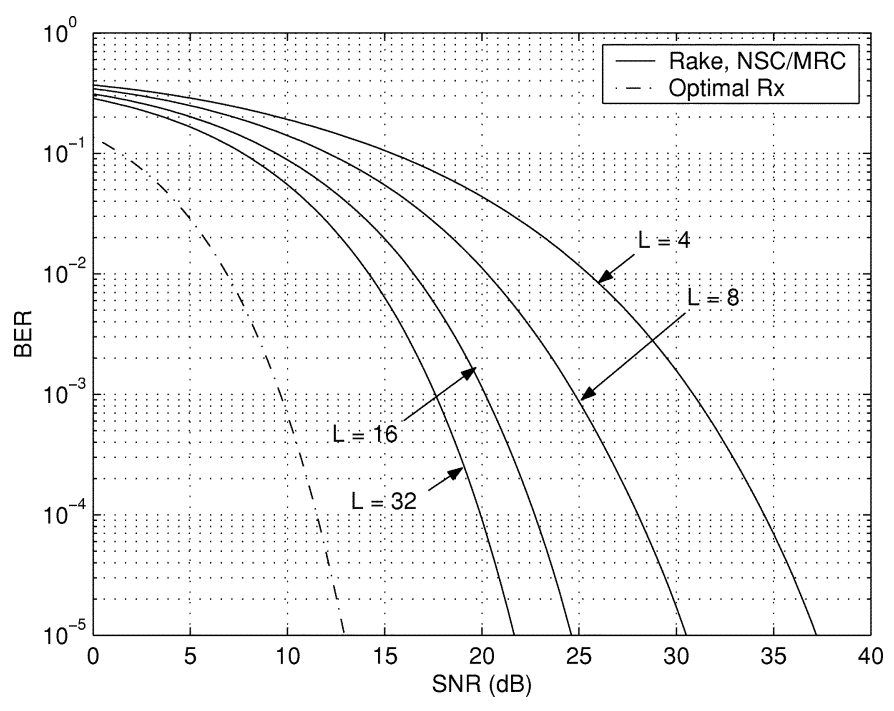

Fig. 5. Performance of binary block-coded overlapped PPM with rake reception and NSC/MRC for $(L=4,8,16,32)$.

tion schemes. In order to generate the numerical results, the exact conditional error probability equation (44) was employed in (43). Fig. 6 reveals the expected cross-over behavior among the performance curves as a result of the noise-on-noise term arising in SLC. In general, there exists an optimal $L$ which minimizes the bit error probability for a given SNR [46]. The performance gains of SC/SLC diminish after about $L=16$ where it suffers a 9.6-dB loss compared with the optimal receiver at $\mathrm{BER}=10^{-5}$. For the case of OOK with SC-NSC/SLC (Fig. 7), there exists only a $1.5-\mathrm{dB}$ gain in performance at $\mathrm{BER}=10^{-5}$ by increasing the number of combined paths from $L=2$ to $L=32$. More significant performance improvement as a function of receiver complexity is demonstrated by the NSC/SLC scheme (Fig. 8) although similar to the case of NSC/MRC, significant performance degradations result for small $L$.

The results of these figures (Fig. 2-8), as well as additional performance results involving binary block-coded OOK with MRC, are provided in Table I. The table indicates that binary 


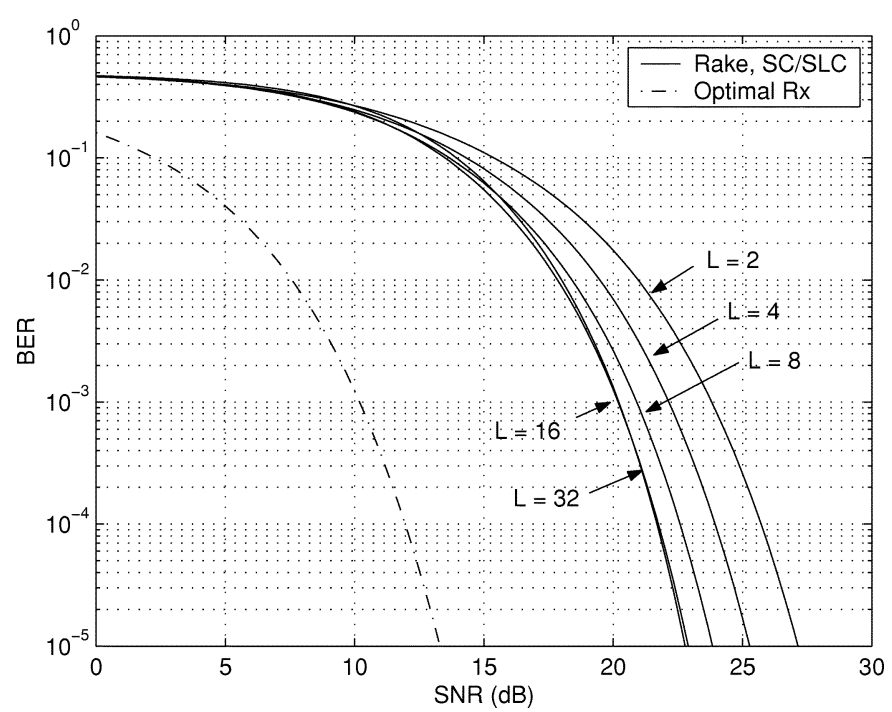

Fig. 6. Performance of binary block-coded OOK with a rake receiver and SC/SLC for $L=2,4,8,16,32$.

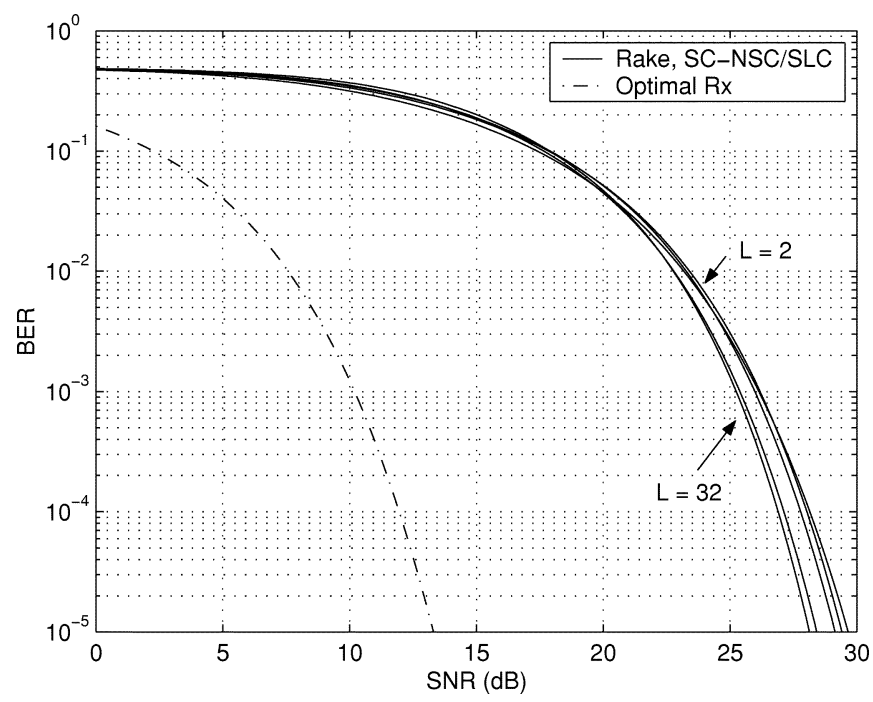

Fig. 7. Performance of binary block-coded OOK with a rake receiver and SC-NSC/SLC for $L=2,4,8,16,32$.

block-coded OOK with MRC suffers approximately a 2-3-dB loss at BER $=10^{-5}$ when compared with overlapped PPM for a particular $L$ and selection scheme. This loss is more consistent with the performance degradation exhibited by OOK relative to overlapped PPM in the AWGN channel. In addition, when comparing the performance of OOK and the various selection schemes with SLC to that with MRC, it is evident that the performance loss of SLC is marginal (approximately $1 \mathrm{~dB}$ at $\mathrm{BER}=10^{-5}$ ) for small $L$ but increases to approximately $3-4 \mathrm{~dB}$ at BER $=10^{-5}$ as $L$ increases. It is noted that the performance of OOK with SLC at $L=64$ and $L=100$ can be approximated by employing the Gaussian approximation (21).

\section{TR System With Autocorrelation Receiver}

We specify the ideal bandpass filter to have center frequency $f_{c}=1.05 \mathrm{GHz}$ and bandwidth $W=2 \mathrm{GHz}$, which corresponds to approximately the $-20 \mathrm{~dB}$ bandwidth of $\tilde{p}(t)$. Although $W$ represents an important design parameter in the performance of

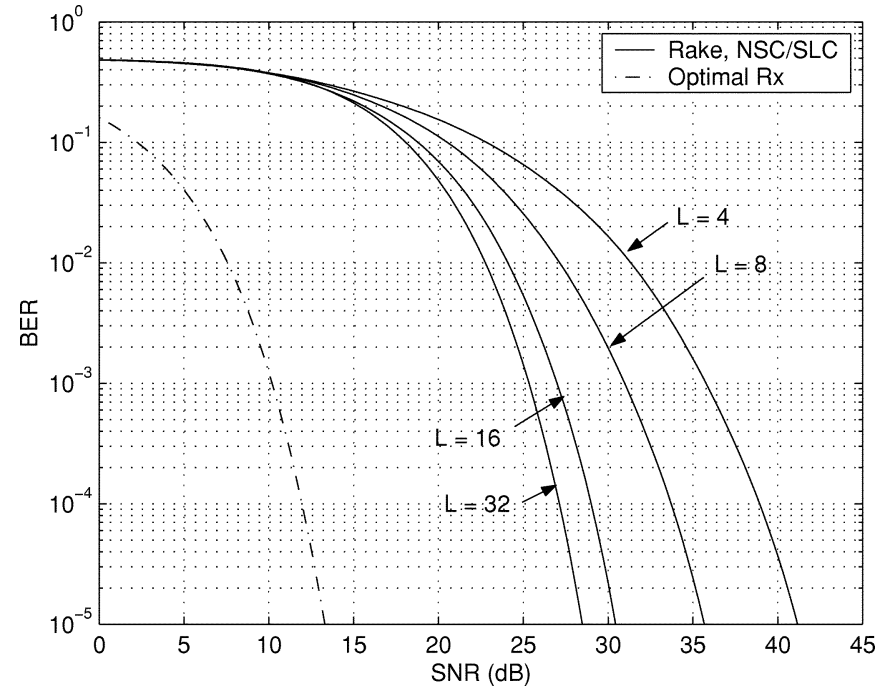

Fig. 8. Performance of binary block-coded OOK with a rake receiver employing NSC/SLC for $L=4,8,16,32$.

autocorrelation receivers, the filter bandwidth is not optimized for the ensuing performance results. In general, increasing $W$ improves the energy capture of the autocorrrelation receiver at the expense of increasing the noise present in the demodulation process.

The TR system with binary block-coded overlapped PPM and autocorrelation reception, where the reference pulses are averaged, suffers a 5.39-dB loss ( $3 \mathrm{~dB}$ of which is accounted for by the transmission of reference pulses) when compared with the optimal receiver at BER $=10^{-5}$ in the AWGN channel (see Fig. 2), [49]. Likewise, the TR system with block-coded OOK suffers a 4.71-dB loss relative to the optimal receiver for block-coded OOK in AWGN (see Fig. 2). The overlapped PPM schemes yields a $1.71-\mathrm{dB}$ gain over OOK at $\mathrm{BER}=10^{-5}$.

The performance of the TR system in the measured multipath channels is detailed in Fig. 9 for various $T$. For the bandpass filter specified, we observe that the performance gains associated with increasing the integration time and equivalently, the energy capture of the autocorrelation receiver, begin to diminish after $T=20 \mathrm{~ns}$. The performance of overlapped PPM and OOK are 11.73 and $11.64 \mathrm{~dB}$, respectively, removed from the performance of the optimal receiver at $\mathrm{BER}=10^{-5}$ when $T=40 \mathrm{~ns}$.

When comparing the performance of the rake receivers in the previous subsection with that of the autocorrelation receiver, the fact that the TR system incurs a 3-dB loss for the transmission of reference pulses must be taken into account since all combining schemes which employ MRC or SC require pilot symbols to perform channel estimation. Despite the penalty, we observe that for the case of binary block-coded overlapped PPM, the autocorrelation receiver for $T=40 \mathrm{~ns}$ performs slightly better than SC/MRC with $L=1$, SC-NSC/MRC with $L=4$, and approximately the same as NSC/MRC with $L=16$. Similarly, the autocorrelation receiver for OOK and $T=40 \mathrm{~ns}$ performs slightly better than SC/MRC with $L=2$, SC-NSC/MRC with $L=16$, NSC/MRC with $L=16$, and SC/SLC with $L=4$.

Although autocorrelation receivers capture a significant portion of the received signal energy, their performance is ultimately limited by the presence of the noise-on-noise term. The 
TABLE I

Performance of Binary Block-Coded Modulation Schemes with Rake Reception AND DIVERSITY COMBINING

\begin{tabular}{|c|c|c|c|c|c|c|c|c|c|}
\hline \multirow{3}{*}{ Mod } & \multirow{3}{*}{$\begin{array}{c}\text { Diversity } \\
\text { Combining } \\
\text { Scheme }\end{array}$} & \multicolumn{8}{|c|}{ Minimum Required SNR (dB) for BER $=10^{-5}$} \\
\hline & & \multicolumn{8}{|c|}{ Combined Multipath Components $(L)$} \\
\hline & & 1 & 2 & 4 & 8 & 16 & 32 & 64 & 100 \\
\hline \multirow{3}{*}{$\begin{array}{c}\text { Overlapped } \\
\text { PPM }\end{array}$} & SC/MRC & 26.37 & 23.66 & 21.22 & 19.14 & 17.51 & 16.56 & 16.23 & 16.18 \\
\hline & SC-NSC/MRC & 26.37 & 26.03 & 25.18 & 24.24 & 22.7 & 21.56 & 21.09 & 20.99 \\
\hline & NSC/MRC & - & - & 37.21 & 30.51 & 24.61 & 21.67 & 20.52 & 20.36 \\
\hline \multirow[t]{3}{*}{$\overline{\overline{\text { OOK }}}$} & $\overline{\text { SC/MRC }}$ & 28.94 & 26.21 & 23.76 & 21.64 & 19.93 & 18.92 & 18.54 & 18.48 \\
\hline & SC-NSC/MRC & 28.94 & 28.68 & 27.86 & 26.85 & 25.32 & 24.11 & 23.58 & 23.46 \\
\hline & NSC/MRC & - & - & 39.18 & 33.0 & 27.21 & 24.34 & 23.13 & 22.92 \\
\hline \multirow[t]{3}{*}{$\overline{\mathrm{OOK}}$} & SC/SLC & - & 27.15 & 25.28 & 23.84 & 22.91 & 22.78 & - & - \\
\hline & SC-NSC/SLC & - & 29.66 & 29.41 & 29.14 & 28.42 & 28.13 & - & - \\
\hline & NSC/SLC & - & - & 41.17 & 35.67 & 30.44 & 28.48 & - & - \\
\hline
\end{tabular}

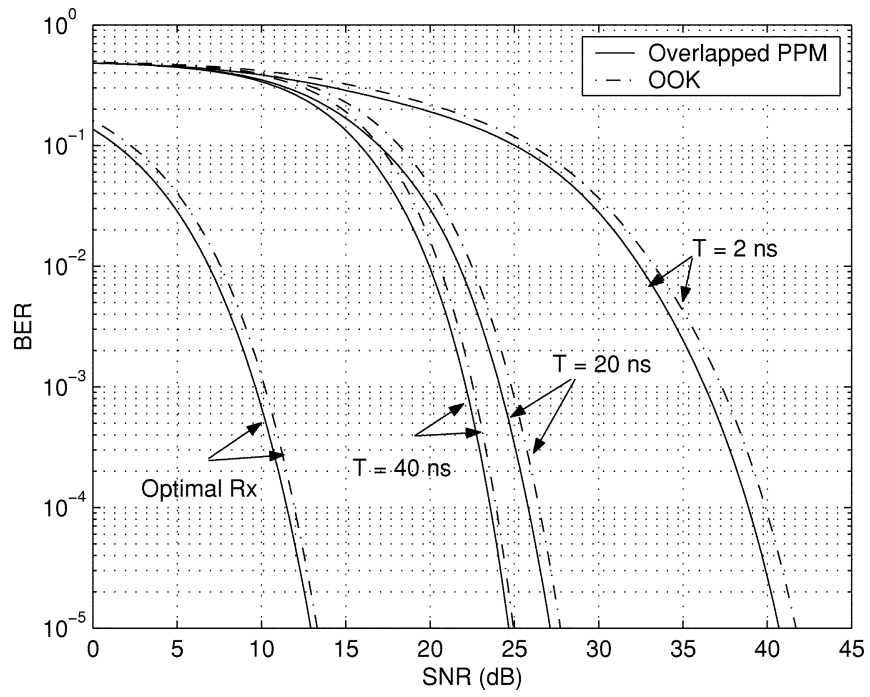

Fig. 9. Performance of TR system with binary block-coded overlapped PPM and OOK and autocorrelation receiver which averages the reference pulses for $T=2,20,40 \mathrm{~ns}$.

suppression of noise by averaging previously received reference pulses requires the implementation of precise delays which may be burdensome. Hence, the signaling scheme must be appropriately designed to reduce the need for noise suppression.

\section{CONCLUSION}

The tradeoff between performance and complexity for rake and autocorrelation receivers is examined for the case of a single-user UWB system employing binary blocked-coded PPM. Numerical results are obtained for a variety of cases by evaluating the analytical derivations with indoor channel measurements.

In order to obtain robust performance, the complexity of rake and autocorrelation receivers may need to be increased. For rake receivers, this implies the use of more fingers and some form of selection combining where strong paths are tracked. As for autocorrelation receivers, the need for noise suppression techniques depends significantly upon the signaling scheme implemented. Future areas of research include the performance of these suboptimal receivers when channel impairments such as narrowband and wideband jamming, intersymbol interference, and multiuser interference are present.

\section{APPENDIX A \\ RAKE RECEIVER WITH SLC}

The derivation of the conditional probability of error for binary block-coded orthogonal PPM with rake reception and SLC follows immediately from [46]. Although the derivation is straightforward, the resulting equations must be evaluated using numerical integration for most cases. As a result, we do not provide the general derivation and only consider the specific case in which $Z_{1}$ in (18) is a conditionally central chi-square random variable $\left(\tau_{p} \geq T_{g}\right)$ and $L$ is even. Under these conditions, the conditional probability of error is given by [51]

$$
\begin{aligned}
P_{e}(\underline{u}) & =\exp \left(-\frac{N_{p} E_{p} \sum_{l=0}^{L-1} \alpha^{2}\left(\tau_{l}\right)}{2 N_{0}}\right) \sum_{l=0}^{\frac{L}{2}-1}\left(\frac{1}{2}\right)^{\frac{L}{2}+l} \\
& \cdot \sum_{k=0}^{l}\left(\begin{array}{c}
l+\frac{L}{2}-1 \\
l-k
\end{array}\right) \frac{1}{k !}\left(\frac{N_{p} E_{p} \sum_{l=0}^{L-1} \alpha^{2}\left(\tau_{l}\right)}{2 N_{0}}\right)^{k} .
\end{aligned}
$$

It is noted that (44) represents the exact conditional probability of error while (21) serves an approximation for large $L$.

\section{APPENDIX B}

\section{TR SYSTEM WITH AUTOCORRELATION RECEIVER}

The conditional expectation of the decision statistic $Z_{\Delta}$ is given by

$$
E\left[Z_{\Delta} \mid \underline{U}=\underline{u}\right]=E\left[Y_{1}+Y_{2}+Y_{3} \mid \underline{U}=\underline{u}\right]+E\left[Y_{4}\right] .
$$

We observe that $Y_{2}$ and $Y_{3}$ (36) are conditionally zero mean Gaussian random variables because $\hat{n}(t)(26)$ is a zero mean random process.

The expected value of $Y_{4}(36)$ is given by

$$
\begin{aligned}
E\left[Y_{4}\right]= & \sum_{j=0}^{N_{p} / 2-1} \sum_{k=0}^{N-1} E\left[X_{4, j, k}\right] \\
= & \frac{T}{N} \sum_{k=0}^{N-1} \sum_{j=0}^{N_{p} / 2-1} R_{\hat{n}}\left(k T_{f}+T_{g}+c_{0, j} \tau_{p}\right) \\
& -R_{\hat{n}}\left(k T_{f}+T_{g}+c_{1, j} \tau_{p}\right) \\
= & 0
\end{aligned}
$$


where $R_{\hat{n}}(\tau)$ is the autocorrelation function of the bandpass-filtered AWGN and the simplification in (48) results from the previous assumptions regarding the code elements $\left\{c_{i, j}\right\}$. As a result, the conditional mean of $Z_{\Delta}$ is simply $Y_{1}$ which is provided in (37).

Next, the conditional variance of the decision statistic $Z_{\Delta}$ is given by

$$
\begin{aligned}
\operatorname{Var}\left(Z_{\Delta} \mid \underline{U}=\underline{u}\right) & =E\left[\left(Y_{2}+Y_{3}+Y_{4}\right)^{2} \mid \underline{U}=\underline{u}\right] \\
& =\sum_{m=2}^{4} \sum_{m^{\prime}=2}^{4} E\left[Y_{m} Y_{m^{\prime}} \mid \underline{U}=\underline{u}\right] .
\end{aligned}
$$

The ensuing derivations employ the assumption $W \gg 1 / T_{g}$ and neglect those terms for which $R_{\hat{n}}(\tau)$ is very small relative to other terms [37], [43].

Employing the definition provided in (35) and (36), we first compute the conditional variance of $Y_{2}$ as follows:

$$
\begin{aligned}
E\left[Y_{2}^{2} \mid \underline{U}=\underline{u}\right] & =\sum_{j=0}^{N_{p} / 2-1} \sum_{j^{\prime}=0}^{N_{p} / 2-1} E\left[X_{2, j} X_{2, j^{\prime}} \mid \underline{U}=\underline{u}\right] \\
& \simeq \sum_{j=0}^{N_{p} / 2-1} E\left[X_{2, j}^{2} \mid \underline{U}=\underline{u}\right] \\
& =\frac{N_{p} E_{p} N_{0}}{2} \zeta_{2}
\end{aligned}
$$

where

$\zeta_{2}=\frac{2}{N_{0}} \int_{0}^{T} \int_{0}^{T}\left[R_{\hat{n}}(t-v)-R_{\hat{n}}\left(t-v+\tau_{p}\right)\right]$ $\cdot \hat{g}(t) \hat{g}(v) d t d v$.

The conditional variance of $Y_{3}$, which is defined in (35) and (36), is given by

$$
\begin{aligned}
E & {\left[Y_{3}^{2} \mid \underline{U}=\underline{u}\right] } \\
& =\sum_{j=0}^{N_{p} / 2-1} \sum_{j^{\prime}=0}^{N_{p} / 2-1} \sum_{k=0}^{N-1} \sum_{k^{\prime}=0}^{N-1} E\left[X_{3, j, k} X_{3, j^{\prime}, k^{\prime}} \mid \underline{U}=\underline{u}\right] .
\end{aligned}
$$

For $W \gg 1 / T_{g}$, the expectation in (55) can be approximated as

$$
\begin{aligned}
& E\left[X_{3, j, k} X_{3, j^{\prime}, k^{\prime}} \mid \underline{U}=\underline{u}\right] \\
& \simeq \delta_{j-j^{\prime}, k-k^{\prime}} \frac{E_{p}}{N^{2}} \\
& \quad \times \int_{0}^{T} \int_{0}^{T}\left[\hat{g}(t)-\hat{g}\left(t+\left(c_{1, j}-c_{0, j}\right) \tau_{p}\right)\right] \\
& \quad \cdot\left[\hat{g}(v)-\hat{g}\left(v+\left(c_{1, j^{\prime}}-c_{0, j^{\prime}}\right) \tau_{p}\right)\right] R_{\hat{n}}(t-v) d t d v
\end{aligned}
$$

where $\delta_{a, b}$ is the Kronecker delta function. By substituting (56) into (55), we obtain the following:

$$
E\left[Y_{3}^{2} \mid \underline{U}=\underline{u}\right] \simeq \begin{cases}\frac{N_{p} E_{p} N_{0}}{2} \varepsilon_{3}, & N=1 \\ \frac{N_{p} E_{p} N_{0}}{2} \zeta_{3}, & N=N_{p} / 2\end{cases}
$$

where

$$
\begin{aligned}
\varepsilon_{3}= & \frac{1}{N_{0}} \int_{0}^{T} \int_{0}^{T} R_{\hat{n}}(t-v)\left[\hat{g}(t) \hat{g}(v)-\hat{g}(t) \hat{g}\left(v+\tau_{p}\right)\right. \\
& -\hat{g}(t) \hat{g}\left(v-\tau_{p}\right)+\frac{1}{2} \hat{g}\left(t+\tau_{p}\right) \hat{g}\left(v+\tau_{p}\right) \\
& \left.+\frac{1}{2} \hat{g}\left(t-\tau_{p}\right) \hat{g}\left(v-\tau_{p}\right)\right] d t d v
\end{aligned}
$$

and

$$
\begin{aligned}
\zeta_{3}= & \frac{2}{3 N_{0}} \int_{0}^{T} \int_{0}^{T} R_{\hat{n}}(t-v)\left[\hat{g}(t) \hat{g}(v)-\hat{g}(t) \hat{g}\left(v+\tau_{p}\right)\right. \\
& -\hat{g}(t) \hat{g}\left(v-\tau_{p}\right)+\frac{1}{4} \hat{g}\left(t+\tau_{p}\right) \hat{g}\left(v+\tau_{p}\right)+\frac{1}{4} \hat{g}\left(t-\tau_{p}\right) \\
& \left.. \hat{g}\left(v-\tau_{p}\right)+\frac{1}{2} \hat{g}\left(t+\tau_{p}\right) \hat{g}\left(v-\tau_{p}\right)\right] d t d v
\end{aligned}
$$

It is noted that averaging the previous $N_{p} / 2$ reference pulses does not yield a significant reduction in the conditional variance of $Y_{3}$ when compared with case of no averaging.

Next, we derive the variance of $Y_{4}$, which is specified in (35) and (36), and initially obtain the equation

$$
E\left[Y_{4}^{2}\right]=\sum_{j=0}^{N_{p} / 2-1} \sum_{j^{\prime}=0}^{N_{p} / 2-1} \sum_{k=0}^{N-1} \sum_{k^{\prime}=0}^{N-1} E\left[X_{4, j, k} X_{4, j^{\prime}, k^{\prime}}\right] .
$$

The expectation inside the summations above can be approximated as

$$
\begin{aligned}
E\left[X_{4, j, k} X_{4, j^{\prime}, k^{\prime}}\right] \simeq & \frac{2}{N^{2}} \delta_{j, j^{\prime}} \delta_{k, k^{\prime}} \int_{0}^{T} \int_{0}^{T} R_{\hat{n}}^{2}(t-v) \\
& -R_{\hat{n}}(t-v) R_{\hat{n}}\left(t-v+\tau_{p}\right) d t d v
\end{aligned}
$$

where we have employed the fact that the expectation of the product of zero mean, jointly Gaussian random variables $\left\{\hat{n}\left(t_{1}\right), \hat{n}\left(t_{2}\right), \hat{n}\left(t_{3}\right), \hat{n}\left(t_{4}\right)\right\}$ is given by [37], [52]

$$
\begin{aligned}
& E\left[\hat{n}\left(t_{1}\right) \hat{n}\left(t_{2}\right) \hat{n}\left(t_{3}\right) \hat{n}\left(t_{4}\right)\right] \\
& \quad=R_{\hat{n}}\left(t_{1}-t_{2}\right) R_{\hat{n}}\left(t_{3}-t_{4}\right)+R_{\hat{n}}\left(t_{1}-t_{3}\right) R_{\hat{n}}\left(t_{2}-t_{4}\right) \\
& \quad+R_{\hat{n}}\left(t_{1}-t_{4}\right) R_{\hat{n}}\left(t_{2}-t_{3}\right) .
\end{aligned}
$$

Substituting the (61) back into (60), we obtain

$$
E\left[Y_{4}^{2}\right] \simeq \begin{cases}\frac{N_{p}}{2} N_{0}^{2} T W \zeta_{4}, & N=1, \\ N_{0}^{2} T W \zeta_{4}, & N=N_{p} / 2\end{cases}
$$

where

$$
\begin{array}{r}
\zeta_{4}=\frac{2}{N_{0}^{2} T W} \int_{0}^{T} \int_{0}^{T} R_{\hat{n}}^{2}(t-v)-R_{\hat{n}}(t-v) \\
\cdot R_{\hat{n}}\left(t-v+\tau_{p}\right) d t d v .
\end{array}
$$

It is evident from (63) that averaging the reference pulses leads to a significant reduction in the variance of the noise-on-noise term whenever $N_{p}$ is large. For the simple case in which $N_{p}=$ 2 , the conditional variance of this term is the same as that when $N_{p} \neq 2$ and averaging is employed over the previous $N_{p} / 2$ reference pulses. However, the Gaussian approximation may no longer hold for $N_{p}=2$ (depending upon $T W$ ) and may overestimate the conditional probability of error for the SNR range of interest.

In order to complete the computation of the conditional variance, we must determine the expectation of the cross terms in 
(50). We note that both $E\left[Y_{2} Y_{4} \mid \underline{U}=\underline{u}\right]$ and $E\left[Y_{3} Y_{4} \mid \underline{U}=\underline{u}\right]$ equal zero because the expected value of the product of three zero mean, jointly Gaussian random variables is zero [37], [52]. In addition, we neglect the term $E\left[Y_{2} Y_{3} \mid \underline{U}=\underline{u}\right]$ for $W \gg$ $1 / T_{g}$.

Summarizing the previous results, the conditional variance of the decision statistic (50) for the case of $N=N_{p} / 2$ is approximated as

$$
\operatorname{Var}\left(Z_{\Delta} \mid \underline{U}=\underline{u}\right) \simeq \frac{N_{p} E_{p} N_{0}}{2}\left[\zeta_{2}+\zeta_{3}+\frac{2 N_{0} T W \zeta_{4}}{N_{p} E_{p}}\right]
$$

\section{APPENDIX C \\ TR SYSTEM WITH AUTOCORRELATION RECEIVER, OOK}

The derivation of the conditional probability of error for the special case of block-coded orthogonal PPM or OOK (40) is obtained by substituting $\tau_{p}=T_{g}$ into the definition of $\rho\left(\tau_{p}\right)$ specified in (37), as well as in (54), (59), and (64). The conditional variance terms then simplify as follows:

$$
\begin{aligned}
\zeta_{2}^{\prime} & \simeq \frac{2}{N_{0}} \int_{0}^{T} \int_{0}^{T} R_{\hat{n}}(t-v) \hat{g}(t) \hat{g}(v) d t d v \\
\zeta_{3}^{\prime} & =\frac{2}{3 N_{0}} \int_{0}^{T} \int_{0}^{T} R_{\hat{n}}(t-v) \hat{g}(t) \hat{g}(v) d t d v \\
\zeta_{4}^{\prime} & \simeq \frac{2}{N_{0}^{2} T W} \int_{0}^{T} \int_{0}^{T} R_{\hat{n}}^{2}(t-v) d t d v
\end{aligned}
$$

and the mean and variance of the decision statistic become

$$
\begin{aligned}
& E\left[Z_{\Delta} \mid \underline{U}=\underline{u}\right]=\frac{N_{p} E_{p}}{2} \int_{0}^{T} \hat{g}^{2}(t) d t \\
& \operatorname{Var}\left(Z_{\Delta} \mid \underline{U}=\underline{u}\right] \simeq \frac{N_{p} E_{p} N_{0}}{2}\left[\zeta_{2}^{\prime}+\zeta_{3}^{\prime}+\frac{2 N_{0} T W \zeta_{4}^{\prime}}{N_{p} E_{p}}\right] .
\end{aligned}
$$

\section{ACKNOWLEDGMENT}

The authors would like to thank R. Scholtz and J. Lee of the University of Southern California, as well as M. Win of MIT, for generously providing the channel data. They would also like to thank members of the UWB research community and former or current colleagues at the University of Michigan for their helpful suggestions and insights. In particular, they are grateful to R. Hoctor, T. Barrett, S. Yano, D. Hall, P. Withington, T. Nolan, T. Ji, D. Yoo, J. Kang, and J. East for their time and assistance. Furthermore, the authors thank the reviewers and editors for their comments.

\section{REFERENCES}

[1] J. D. Taylor, Introduction to Ultra-Wideband Systems. Ann Arbor, MI: CRC Press, 1995.

[2] L. Y. Astanin and A. A. Kostylev, Ultrawideband Radar Measurements Analysis and Processing. London, U.K.: IEE, 1997.

[3] "Revision of Part 15 of the Commission's Rules Regarding Ultra-Wideband Transmission," Federal Communications Commission, 1st Rep. and Order, 2002.

[4] T. W. Barrett, "History of ultra wideband communications and radar: Part I, UWB communications," Microw. J., pp. 22-56, Jan. 2001.

[5] M. Z. Win and R. A. Scholtz, "Impulse radio: How it works," IEEE Commun. Lett., vol. 2, pp. 36-38, Feb. 1998.

[6] R. A. Scholtz, "Multiple access with time hopping impulse modulation," in Proc. 1993 MILCOM, 1993, pp. 447-450.
[7] M. Z. Win and R. A. Scholtz, "Ultra-wide bandwidth time-hopping spread-spectrum impulse radio for wireless multiple-access communications," IEEE Trans. Commun., vol. 48, pp. 679-689, Apr. 2000.

[8] F. Ramirez-Mireles, "Performance of ultrawideband SSMA using time hopping and $M$-ary PPM," IEEE J. Select. Areas Commun., vol. 19, pp. 1186-1196, 2001.

[9] R. T. Hoctor and H. W. Tomlinson, "An overview of delay-hopped, transmitted-reference RF communications," in Technical Information Series: G.E. Research and Development Center, Jan. 2002, pp. 1-29.

[10] J. R. Foerster, "The performance of a direct sequence spread ultra wideband system in the presence of multipath, narrowband interference, and multiuser interference," in Proc. 2002 UWBST, 2002, pp. 87-92.

[11] R. T. Hoctor and H. W. Tomlinson, "Delay-hopped transmited-reference RF communications," in Proc. 2002 UWBST, 2002, pp. 265-270.

[12] B. M. Sadler and A. Swami, "On the performance of UWB and DS-spread spectrum," in Proc. 2002 UWBST, 2002, pp. 289-292.

[13] J. D. Choi and W. E. Stark, "Performance analysis of ultra-wideband spread-spectrum communications in narrowband interference," in Proc. 2002 MILCOM, 2002.

[14] R. A. Scholtz et al., "UWB radio deployment challenges," in Proc. 2000 PIMRC, 2000, pp. 620-625.

[15] M. Z. Win, R. A. Scholtz, and M. A. Barnes, "Ultra-wide bandwidth signal propagation for indoor wireless communications," in Proc. 1997 ICC, 1997, pp. 56-60.

[16] M. Z. Win and R. A. Scholtz, "On the energy capture of ultrawide bandwidth signals in dense multipath environments," IEEE Commun. Lett., vol. 2, pp. 245-247, Sept. 1998.

[17] R. J. Cramer, M. Z. Win, and R. A. Scholtz, "Impulse radio multipath characteristics and diversity reception," in Proc. 1998 ICC, 1998, pp. $1650-1654$.

[18] R. J. Cramer, "An Evaluation of Ultra-Wideband Propagation Channels," Ph.D. dissertation, Univ. Southern California, Los Angeles, 2000.

[19] R. J. Cramer, R. A. Scholtz, and M. Z. Win, "On the analysis of uwb communication channels," in Proc. 1999 MILCOM, 1999, pp. $1191-1195$.

[20] M. Z. Win and R. A. Scholtz, "On the robustness of ultra-wide bandwidth signals in dense multipath environments," IEEE Commun. Lett., vol. 2, pp. 51-53, Feb. 1998.

[21] F. Ramirez-Mireles, "On performance of ultra wideband signals in Gaussian noise and dense multipath," IEEE Trans. Veh. Technol., vol. 50, pp. 244-249, Jan. 2001.

[22] J. D. Taylor, Ultra-Wideband Radar Technology. New York: CRC Press, 2001

[23] R. J. Cramer, R. A. Scholtz, and M. Z. Win, "Evaluation of an ultrawide-band propagation channel," IEEE Trans. Antennas Propagat., vol. 50, pp. 561-570, May 2002.

[24] R. J. Fontana, J. F. Larrick, and J. E. Cade, "An ultra-wideband communication link for unmanned vehicle applications," in Proc. 1997 AUVSI, 1997.

[25] J. W. Toevs et al., "UWB radar for ground vehicle self protect," in Proc. 1992 MTT Symp., 1992, pp. 1491-1494.

[26] C. L. Bennett and G. F. Ross, "Time-domain electromagnetics and its applications," Proc. IEEE, vol. 66, pp. 299-318, Mar. 1978.

[27] F. Ramirez-Mireles, M. Z. Win, and R. A. Scholtz, "Performance of ultra-wideband time shift-modulated signals in the indoor wireless impulse radio channel," in Proc. 1998 Asilomar Conf., 1998, pp. 192-196.

[28] F. Ramirez-Mireles and R. A. Scholtz, "Performance of equicorrelated ultra-wideband pulse-position-modulated signals in the indoor wireless impulse radio channel," in Proc. 1997 PACRIM, 1997, pp. 640-644.

[29] J. R. Foerster, "The effects of multipath interference on the performance of UWB systems in an indoor wireless channel," in Proc. 2001 Spring Vehicular Technology Conf., 2001, pp. 1176-1180.

[30] D. Cassioli et al., "Performance of low-complexity rake reception in a realistic UWB channel," in Proc. 2002 ICC, 2002, pp. 763-767.

[31] A. Taha and K. Chugg, "Multipath diversity reception of wireless multiple access time-hopping digital impulse radio," in Proc. 2002 UWBST, 2002, pp. 283-288.

[32] N. van Stralen et al., "Delay-hopped transmitted-reference experimental results," in Proc. 2002 UWBST, 2002, pp. 93-98.

[33] T. Eng, N. Kong, and L. B. Milstein, "Comparison of diversity combining techniques for Rayleigh-fading channels," IEEE Trans. Commun., vol. 44, pp. 1117-1129, Sept. 1996.

[34] M. Alouini and M. K. Simon, "Performance of coherent receivers with hybrid SC/MRC over Nakagami- $m$ fading channels," IEEE Trans. Veh. Technol., vol. 48, pp. 1155-1164, July 1999.

[35] M. Z. Win and J. H. Winters, "Analysis of hybrid selection/maximalratio combining in Rayleigh fading," in Proc. 1999 ICC, 1999, pp. 6-10. 
[36] M. L. Welborn, "System considerations for ultra-wideband wireless networks," in Proc. 2001 RAWCON, 2001, pp. 5-8.

[37] M. K. Simon et al., Digital Communication Techniques. Englewood Cliffs, NJ: Prentice-Hall, 1995

[38] M. K. Simon et al., Spread Spectrum Communications. Rockville, MD: Computer Science, 1985.

[39] Y. B. Okunev, Theory of Phase-Difference Modulation. Moscow, Russia: Svyaz Press, 1979.

[40] Y. B. Okunev and N. M. Sidorov, "Noise immunity of a carrier-frequency-invariant demodulator of DPSK-2 signals," Telecommun. Radio Eng., no. 6, pp. 81-83, Aug. 1986.

[41] R. E. Gut, "Noise immunity of autocorrelation reception of single PSK signals," Telecommun. Radio Eng., no. 10, pp. 120-122, 1972.

[42] R. E. Gut, Y. B. Okunev, and N. M. Sidorov, "Effect of carrier detuning on the noise immunity of autocorrelation reception of DPSK signals," Telecommun. Radio Eng., no. 9, pp. 96-98, 1987.

[43] J. H. Park, "On binary DPSK detection," IEEE Trans. Commun., vol. COM-26, pp. 484-486, Apr. 1978.

[44] M. Z. Win, "Spectral density of random time-hopping spread spectrum UWB signals with uniform timing jitter," IEEE Commun. Lett., vol. 2, pp. 36-38, Feb. 1998

[45] J. D. Choi and W. E. Stark, "Performance analysis of rake receivers for ultra-wideband communications with PPM and OOK in multipath channels," in Proc. 2002 ICC, 2002, pp. 1969-1973.

[46] J. G. Proakis, Digital Communications. New York: McGraw-Hill, 1995.

[47] W. C. Jakes, Microwave Mobile Communications. New York, NY: IEEE Press, 1974.

[48] D. Cassioli, M. Z. Win, and A. F. Molish, "The ultra-wide bandwidth channel: From statistical model to simulations," IEEE J. Select. Areas Commun., vol. 20, pp. 1247-1257, Aug. 2002.

[49] J. D. Choi and W. E. Stark, "Performance of autocorrelation receivers for ultra-wideband communications with PPM in multipath channels," in Proc. 2002 UWBST, 2002, pp. 213-218.

[50] S. S. Ghassenizadeh et al., "A statistical path loss model for in-home UWB channels," in Proc. 2002 UWBST, 2002, pp. 59-64.

[51] W. E. Stark, Digital Communication Theory. Ann Arbor, MI: Univ. Michigan, 2000.

[52] H. Stark and J. W. Woods, Probability, Random Processes, and Estimation Theory for Engineers. Englewood Cliffs, NJ: Prentice-Hall, 1994.

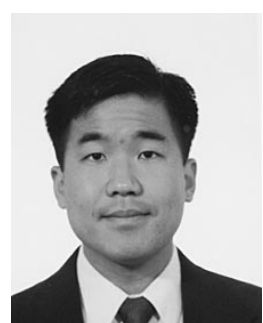

John D. Choi (S'95) received the B.S.E.E. degree from Duke University, Durham, NC, in 1997, and the M.S.E.E. degree from the University of Michigan, Ann Arbor, in 1998, where he is currently working toward the Ph.D. degree.

His research interests include communication theory and networks.

Mr. Choi is a Member of Phi Beta Kappa, Tau Beta $\mathrm{Pi}$, and Eta Kappa $\mathrm{Nu}$.

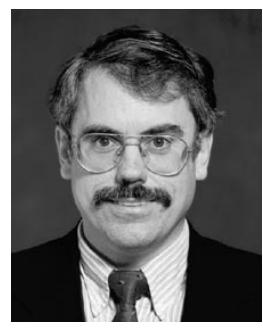

Wayne E. Stark (S'77-M'78-SM'94-F'98) received the B.S. degree (highest honors), and the M.S. and Ph.D. degrees in electrical engineering from the University of Illinois, Urbana-Champaign, in 1978,1979 , and 1982, respectively.

Since September 1982, he has been a Faculty Member in the Department of Electrical Engineering and Computer Science, University of Michigan, Ann Arbor, where he is currently a Professor. His research interests include the areas of coding and communication theory, especially for spread-spectrum and wireless communications networks.

Dr. Stark is a Member of Eta Kappa Nu, Phi Kappa Phi, and Tau Beta Pi. He was involved in the planning and organization of the 1986 International Symposium on Information Theory, held in Ann Arbor, MI. From 1984 to 1989, he was Editor for Communication Theory of the IEEE TRANSACTIONS ON COMMUNICATIONS in the area of spread-spectrum communications. He was selected by the National Science Foundation as a 1985 Presidential Young Investigator. He is Principal Investigator of an Army Research Office Multidisciplinary University Research Initiative (MURI) Project on low-energy mobile communications. 